Gerlach, Katrin; Henning, Günter:

\title{
Patienteninformierung - erste Ansätze auf dem Gebiet des Diabetes mellitus
}

Zuerst erschienen in: Biomedizinische Technik = Biomedical Engineering. Berlin [u.a.] : de Gruyter. - 45 (2000), S1, S. 355-356.

Erstveröffentlichung: 2000

Datum Digitalisierung: 2009-07-17

ISSN (online): $\quad$ 1862-278X

ISSN (print): $\quad$ 0013-5585

DOI: $\quad 10.1515 /$ bmte.2000.45.s1.355

[Zuletzt gesehen: 2019-08-20]

„Im Rahmen der hochschulweiten Open-Access-Strategie für die Zweitveröffentlichung identifiziert durch die Universitätsbibliothek IImenau. "

"Within the academic Open Access Strategy identified for deposition by IImenau University Library."

„Dieser Beitrag ist mit Zustimmung des Rechteinhabers aufgrund einer (DFG-geförderten) Allianz- bzw. Nationallizenz frei

zugänglich."

"This publication is with permission of the rights owner freely accessible due to an Alliance licence and a national licence

(funded by the DFG, German Research Foundation) respectively."

\section{DFG}

Nationallizenzen 


\title{
PATIENTENINFORMIERUNG - ERSTE ANSÄTZE AUF DEM GEBIET DES DIABETES MELLITUS
}

\author{
K. Gerlach, G. Henning \\ TU Ilmenau, Institut für Biomedizinische Technik und Informatik, PF 100 565, 98684 Ilmenau \\ e-mail: katrin.gerlach@informatik.tu-ilmenau.de
}

\section{EINLEITUNG}

An der Stoffwechselkrankheit Diabetes mellitus erkranken neuesten Erhebungen zufolge rund 5\% der Gesamtbevölkerung Deutschlands - sie kann deshalb als Volkskrankheit bezeichnet werden. Betroffen sind davon die unterschiedlichsten Patientengruppen (Kinder, Jugendliche, junge und ältere Erwachsene, Schwangere).

Eine erfolgreiche Therapie des Diabetes mellitus erfordert die aktive Mitarbeit des Patienten, eine umfassende Patienteninformierung ist dafür wichtige Voraussetzung.

Mit Hilfe eines Fragebogens wurde von den Autoren der derzeitige Informienungsstand der Patienten erfasst. Die daraus abgeleiteten Anforderungen für den Informierungsprozess bilden u.a. die Grundlage für die Entwicklung erster Komponenten eines Patienteninformierungssystems (PIS).

\section{MATERIALIEN UND METHODEN}

Kennzeichnend für den Informierungsprozess von Patienten ist die Komplexität des zu vermittelnden medizinischen Wissens und die dadurch auftretenden Kommunikationsprobleme zwischen Fachkraft und Patient. Erschwerend kommt hinzu, dass im medizinischen Bereich die vorhandenen Informationsquellen unstrukturiert, breit gestreut und vom medizinischen Laien meist nicht benutzbar sind. Es stellt sich deshalb die Frage, ob durch die Nutzung moderner Informationstechnologien diese Situation verbessert werden kann.

Beim Einsatz von elektronischen Medien zur Vermittlung von medizinischem Wissen müssen einige methodische Voraussetzungen gegeben sein. Zwei wesentliche Aspekte sollen hier genannt werden:

1. Eine problemspezifische Auswahl der entsprechenden Technologie ist entscheidend für den Erfolg und den Zeitaufwand bei der Informierung.

2. Die Akzeptanz des Informationsangebotes durch die Nutzer hängt von der Repräsentation des vorhandenen medizinischen Wissens unter Beachtung von der Strukturierung des Wissens, Qualität der Informationen, Zielgruppenorientierung, Übersichtlichkeit und sinnvoller Nutzerführung ab.
Zuerst sollte der Stand der Patienteninformierung analysiert werden. Zur Ermittlung des Informierungsverhaltens von Diabetikern wurde ein Fragebogen entwickelt, der in einer speziellen Diabetiker-Zeitschrift [1] und im Internet [2] veröffentlicht wurde.

\section{ERGEBNISSE}

Die Auswertung der 174 eingesandten Fragebögen ergab viele interessante Fakten und warf gleichzeitig neue Fragestellungen auf. Besonders bemerkenswert war, dass der eigene Kenntnisstand $\mathrm{zu} 93 \%$ als gut und besser eingeschätzt wurde, während sich aber insgesamt $52 \%$ der Einsender als nicht gut informiert fühlen.

Die derzeitige Informierungssituation von Diabetikern stellt sich wie folgt dar:

Das medizinische Grundwissen erhält der Patient aus

- regelmäßigen Arzt - Patienten - Konsultationen (meist beim Hausarzt),

- Diabetiker - Schulungen,

- Printmedien.

Zusätzliche Informationsquellen sind

- Facharzt,

- Selbsthilfegruppen,

- Diabetiker - Vereinigungen,

- elektronische Medien, z.B. Internet,

- medizinische Laien.

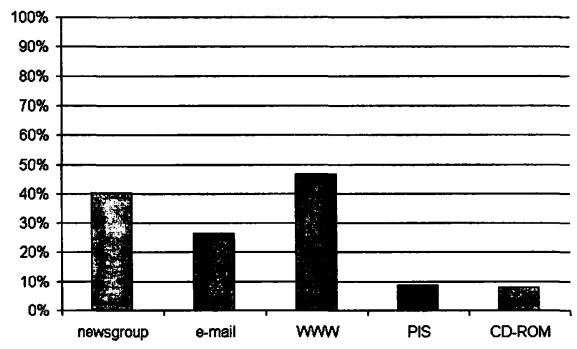

Abb. 1: Nutzung von elektronischen Medien 
Bei der weiteren Analyse zum Informierungsverhalten ergab sich folgendes Bild bei der Nutzung traditioneller Medien (Printmedien, Rundfunk, Fernsehen):

- Nur $2 \%$ der Einsender benutzen diese nicht.

- Die Nutzung der einzelnen Medien ist sehr unterschiedlich, z.B. nutzen $47 \%$ selten oder nie das Fernsehen und $75 \%$ den Rundfunk zur Informierung über ihre Krankheit.

- Von den Printmedien werden Zeitschriften von den Diabetikern bevorzugt, diese werden regelmäßig von über $87 \%$ der befragten Diabetiker gelesen.

Einen Überblick über die Nutzung der elektronischen Medien gibt Abbildung 1, interessante Ergebnisse sind u.a.:

- Mehr als die Hälfte der Einsender setzen heute schon elektronische Medien (WWW, Newsgroups, E-MailListen) ein.

- Patienteninformierungssysteme und CD-ROMAnwendungen werden derzeit kaum genutzt.

- Das Nutzungsverhalten innerhalb der Altersgruppen differiert nicht sehr stark, siehe Abbildung 2.

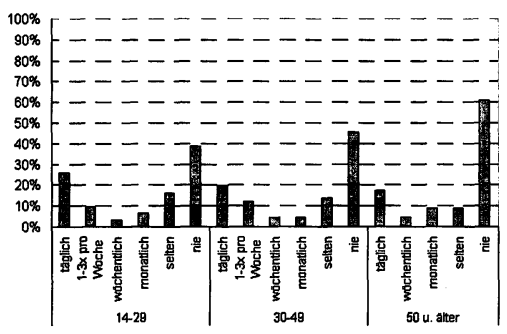

Abb. 2: Nutzung von Newsgroups zur Informierung

Als Plattform für ein künftiges Patienteninformierungssystem wurde ein Web-Angebot entwickelt. Es enthält Informationen zur Diagnose und Therapie des Diabetes mellitus. Wesentliche Bestandteile sind

- Erläuterungen zum Gesundheits-Pass Diabetes,

- kommentierte Linkliste,

- Leitfaden für die Behandlung des nichtinsulinabhängigen Diabetes mellitus.

Abbildung 3 zeigt die Einstiegsseite zum GesundheitsPass Diabetes. Hier erhält der Patient Informationen zur Bedeutung der Eintragungen. Die kommentierte Linkliste dient als Startpunkt für die weitere Informationssuche im Internet; sie unterstützt besonders Einsteiger darin, zu relevanten WWW-Seiten zu gelangen. Ein Beispiel für die mediengerechte Aufarbeitung von medizinischen Dokumenten ist der oben erwähnte Leitfaden.

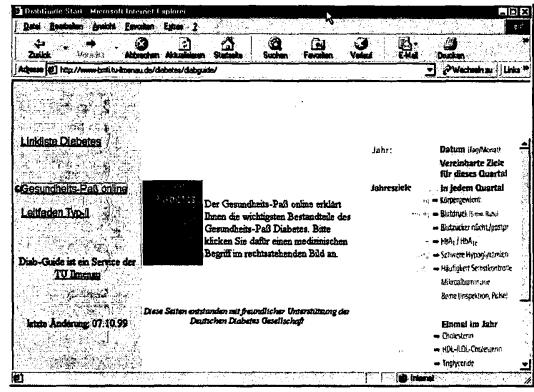

Abb. 3: Web-Seite zur Erläuterung des GesundheitsPasses Diabetes

\section{SCHLUSSFOLGERUNGEN}

Die Auswertung der eingesandten Fragebögen ergab, dass elektronische Medien von vielen Diabetikern zur Informierung über ihre Krankheit eingesetzt werden. Sie stellen eine sinnvolle Ergänzung zu den traditionellen Medien dar. Die von den Autoren im Vortrag zu diskutierende Idee der individuellen Patienteninformierung kann nur in einer Zusammenführung verschiedener Informationsquellen liegen. Um die Compliance zwischen Arzt und Patient zu verbessern, werden Patienteninformierungssysteme in Zukunft stärker eingesetzt werden.

\section{LITERATURHINWEISE}

[1] K. Gerlach, "Wie informieren wir uns", Insuliner, Nr. 47, Kirchen-Freusburg, 1999

[2] Internetadresse: http://www-bmti.tu-ilmenau.de/diabetes/diabguide/

[3] K. Gerlach, G. Henning, D. Westphal, "Electronic information systems for diabetes patients", Computers in Diabetes'98, Barcelona, Diabetes, Nutrition \& Metabolism, vol. 11, n. 1, Milano, 1998

[4] K. Gerlach, G. Henning, A. Kaeding, "Knowledge representation in medicine on the example of the conversation of guidelines for diabetics into a hypertext document" in Proc. of the European Medical \& Biological Engineering Conf. (EMBEC), Part 1, p. 660-661, Wien, 1999

[5] C. O. Köhler, „Patienteninformierung: Eine neue Aufgabe der Ärzte?"“, Deutsches Ärzteblatt - Supplement: Praxis Computer, vol. 40, p. 2, 1998 